\title{
Association between IL-33 and other inflammatory factors in patients with rheumatoid arthritis and in fibroblast-like synoviocytes in vitro
}

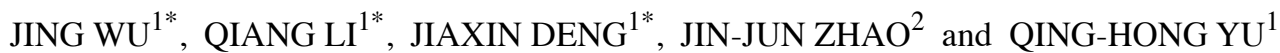 \\ ${ }^{1}$ Rheumatology and Clinical Immunology Department, Zhujiang Hospital, Southern Medical \\ University; ${ }^{2}$ Rheumatology and Clinical Immunology Department, Nanfang Hospital, \\ Southern Medical University, Guangzhou, Guangdong 510282, P.R. China
}

Received May 1,2020; Accepted October 16, 2020

DOI: $10.3892 /$ etm.2020.9592

\begin{abstract}
IL-33 is a member of the IL-1 family of cytokines whose role remains controversial in rheumatoid arthritis (RA). The present study was performed to evaluate the correlation of IL-33 with other cytokines and chemokines in serum and the synovia, and to explore the nature of the association. The concentration of IL-33 in samples from 96 patients with RA was analyzed. The response of fibroblast-like synoviocytes (FLSs) to treatment with different concentrations of IL-33 was assessed in vitro. IL-33 was indicated to exhibit an association with multiple cytokines and chemokines in synovial fluid with an inverted-U-shaped trend, including IL-6, IL-1 $\beta$, IL-8, MIG and IP-10, but not in the serum. Furthermore, in vitro experiments confirmed that IL-33 also exerted a U-type dose-dependent regulatory effect on FLS function. In addition, the data-points do not exactly follow the U-shaped curve fit in most cases, therefore, the applicability of this mathematical model in clinical practice is limited.
\end{abstract}

\section{Introduction}

Rheumatoid arthritis (RA) is a chronic and refractory autoimmune joint disease, characterized by the proliferation

Correspondence to: Dr Qing-Hong Yu, Rheumatology and Clinical Immunology Department, Zhujiang Hospital, Southern Medical University, 253 Gongye Ave, Guangzhou, Guangdong 510282, P.R. China

E-mail: yuqinghong@smu.edu.cn

Dr Jin-Jun Zhao, Rheumatology and Clinical Immunology Department, Nanfang Hospital, Southern Medical University, 1838 Guangzhou Ave, Guangzhou, Guangdong 510282, P.R. China E-mail: zhao7749@126.com

${ }^{*}$ Contributed equally

Key words: IL-33, rheumatoid arthritis, fibroblast-like synoviocytes, cytokines, chemokines of synoviocytes in inflamed synovia and the expression of inflammatory cytokines and chemokines in synoviocytes (1). Multiple pro-inflammatory and inflammatory cytokines have been indicated to participate in the development of RA. A variety of cytokines and chemokines are present in the synovium of patients with RA, which have an important role in the maintenance of the inflammatory response $(2,3)$. Important pro-inflammatory cytokines, including IL-33, IL-1 $\beta$, IL-6, IL-8 and TNF- $\alpha$, and chemokines such as MIG and IP-10, are considered markers for the diagnosis of RA and represent therapeutic targets (3-9). A complex mechanistic network exists between these cytokines and chemokines.

IL-33 is a member of the IL-1 family of cytokines (10). Supporting evidence regarding the association between IL-33 and pathogenesis of RA has been provided by Matsuyama et al (11), who reported that IL-33 levels were elevated in the serum and synovial fluid of patients with RA, demonstrating a positive association with disease activity. IL-33 is expressed in synovial fluid of patients with RA and released from fibroblast-like synoviocytes (FLSs) following stimulation with TNF- $\alpha$ and IL-1 $\beta(11,12)$. IL-33 is able to activate human mast cells to release IL-8, in patients with RA (13). A study indicated that IL-33 was able to enhance the expression of MIG and IP-10 in wild-type mice with colitis (14). IL-33 has an important role and a complex relationship with cytokines and chemokines in RA $(12,15,16)$. However, the role of IL-33 remains controversial, with a number of studies suggesting that it promotes inflammation (17), while others indicate that the response is inhibitory (18). A confounding observation is that high levels of IL-33 may be detected in the serum or synovial fluid of just one third to half of all patients with RA, suggesting that IL-33 is widely variable in RA $(11,19,20)$. A significant number of patients with RA have low levels of IL-33 in serum but still exhibit high disease activity (19). Since contrary results are increasingly observed in RA, the present study hypothesized that the concentrations of IL-33 and other cytokines and chemokines may not be linearly correlated, but a more complex polynomial association should be further investigated. The present study aimed to establish a correlation curve model of cytokines (IL-8, MIG and IP-10) and chemokines (IL-1 $\beta$, TNF- $\alpha$ and IL-6) 
with IL-33 in serum and synovia, and to explore the nature of the relationship.

\section{Materials and methods}

Patient enrollment. A total of 96 patients with RA who had presented at the Rheumatology Clinic of Zhujiang Hospital of Southern Medical University between 1st April 2019 to 1st September 2020 (Guangzhou, China) were recruited for the study. All patients fulfilled the 2010 RA classification criteria (21) and disease activity score DAS28-erythrocyte sedimentation rate $(\mathrm{ESR}) \geq 2.6$. The disease activity was graded according to DAS28-ESR: <2.6 (clinical remission), 2.6-3.1 (low), 3.2-5.0 (moderate activity) and $>5.1$ (high disease activity) (22). Patients with remission of RA were excluded. Serum and synovial fluid samples were collected as appropriate from patients with RA after obtaining written informed consent.

Human FLS culture. Synovial tissue samples were obtained by knee joint arthroscopy from the knees of five patients with active RA, with a DAS28-ESR $>5.1$. All patients with RA fulfilled the 2010 RA classification and signed written informed consent. Synovial tissues were cut into $1-2 \mathrm{~mm}^{3}$ pieces and incubated with collagenase I for $1-3 \mathrm{~h}$ at $37^{\circ} \mathrm{C}$ to isolate synoviocytes (RA-FLSs). Upon reaching 95\% confluency, cells were subsequently digested using $0.25 \%$ trypsin, collected, resuspended and plated for proliferation. Following subculture, RA-FLSs at passages 3-6 were used for subsequent experiments. RA-FLSs were cultured in DMEM (Gibco; Thermo Fisher Scientific, Inc.) containing 10\% FBS (Gibco; Thermo Fisher Scientific, Inc.), $100 \mathrm{U} / \mathrm{ml}$ penicillin and $100 \mu \mathrm{g} / \mathrm{ml}$ streptomycin in a humidified atmosphere containing $5 \% \mathrm{CO}_{2}$ at $37^{\circ} \mathrm{C}$. The culture medium was refreshed every 3-4 days. Cells at passage 3 to 6 were used for the experiments. Individual cell samples from the patients were used in the assays.

Synovial-cell activation. RA-FLSs were seeded in the wells of 24-well plates at a density of $2 \times 10^{4}$ cells/well. After $24 \mathrm{~h}$ of culture, $0,10,50,100$ or $150 \mathrm{ng} / \mathrm{ml}$ human IL-33 (R\&D Systems) was added, followed by incubation for 4 or $24 \mathrm{~h}$ in a humidified atmosphere containing $5 \% \mathrm{CO}_{2}$ at $37^{\circ} \mathrm{C}$. Human IL-17 (100 ng/ml; R\&D Systems) and TNF- $\alpha(100 \mathrm{ng} / \mathrm{ml}$; R\&D Systems), two cytokines known to activate synoviocytes, were used as the positive controls (23), followed by incubation for 4 or $24 \mathrm{~h}$ in a humidified atmosphere containing $5 \% \mathrm{CO}_{2}$ at $37^{\circ} \mathrm{C}$.

Reverse transcription-quantitative PCR (RT-qPCR). Total RNA was isolated from RA-FLSs using TRIzol ${ }^{\circledR}$ reagent (Invitrogen; Thermo Fisher Scientific, Inc.) in accordance with the manufacturer's protocols. RT was conducted using the first-strand cDNA synthesis kit (cat. no. RR047A; Takara Biotechnology Co., Ltd.) in accordance with the manufacturer's protocols. Real-time PCR was performed using a SYBR Premix ExTaq kit (cat. no. RR420A; Takara Biotechnology Co., Ltd.) to assess the expression of IL-6 in accordance with the manufacturer's protocols, IL-8, MIG, IP-10, IL-1 $\beta$ and TNF- $\alpha$. The PCR reactions were performed on an ABI 7500 Real-Time PCR system (Applied Biosystems; Thermo Fisher Scientific, Inc.) in accordance with the manufacturer's protocols. The following thermocycling conditions were used for the qPCR: Initial denaturation $\left(95^{\circ} \mathrm{C}, 30 \mathrm{sec}, 1 \mathrm{cycle}\right)$ and dissociation $\left(95^{\circ} \mathrm{C}\right.$ for $5 \mathrm{sec} ; 60^{\circ} \mathrm{C}$ for $34 \mathrm{sec} ; 40$ cycles). Relative mRNA expression was calculated using the $2^{-\Delta \Delta C q}$ method (24), the $\Delta \Delta \mathrm{Ct}(\Delta \Delta \mathrm{Ct}=\Delta \mathrm{Ct}$ sample- $\Delta \mathrm{Ct}$ control $)$ was used to indicate the ratio of the expression of the target gene in the model group to that of the control group. The primers were synthesized by Sangon Biotech Co., Ltd.. Primer sequences are listed in Table I.

ELISA. The concentrations of cytokines and chemokines in serum and synovial fluid samples were measured using ELISA in accordance with the manufacturer's protocols, including IL-33 (cat. no. D3300B; R\&D Systems, Inc.), IL-1ß (cat. no. DLB50; R\&D Systems, Inc.), TNF- $\alpha$ (cat. no. 88734688; Thermo Fisher Scientific,Inc.), IL-6 (cat. no. 88706688; Thermo Fisher Scientific, Inc.), IL-8 (cat. no. 88808622; Thermo Fisher Scientific, Inc.), MIG (cat. no. EHMIG; Invitrogen; Thermo Fisher Scientific, Inc.) and IP-10 (cat. no. KAC2361; Invitrogen, Thermo Fisher Scientific, Inc.). After stimulation of $2 \times 10^{4}$ RA-FLSs with human IL-33 as described above, the supernatants of the FLS cell cultures were collected and IL-6, IL-8, MIG, IP-10, TNF- $\alpha$ and IL-1 $\beta$ concentrations were quantified. The optical density values of each sample were measured at $450 \mathrm{~nm}$.

Statistical analysis. Statistical analyses were performed using SPSS 24.0 (IBM Corp.) and GraphPad Prism 8.0 (GraphPad Software, Inc.). Values are presented as the mean \pm standard deviation or the median (interquartile range). Differences between groups were analyzed using the Kruskal-Wallis test ( $>2$ groups) or a Mann-Whitney U test (two groups). Mann-Whitney U-test was also used as a post-hoc test after the Kruskal-Wallis test, and Bonferroni correction was performed for these data. Second-order polynomial regression analyses were performed using SPSS 24.0 to quantify the associations among the variables. All statistical tests and confidence intervals were two-sided and $\mathrm{P}<0.05$ was considered to indicate statistical significance.

\section{Results}

Baseline clinical and demographic features. A total of 96 patients with RA were recruited, from whom 40 serum samples ( 35 females and 5 males; mean age, $51.80 \pm 13.37$ years) and 56 synovial samples (43 females and 13 males; mean age, $48.85 \pm 13.39$ years) were obtained. Patients characteristics are displayed in Table II.

Cytokine and chemokine levels in patients with RA. At the baseline, there were no differences in IL-33, MIG and IL-6 concentrations between the synovial and serum samples of patients with RA (Fig. 1A, B and D). The IP-10 concentration was significantly higher in synovial fluid than in serum (Fig. 1C). The IL-8, TNF- $\alpha$ and IL- $\beta$ concentrations were significantly higher in serum than in synovial fluid (Fig. 1E-G).

IL-33 concentration follows an inverted-U-shaped curve in response to cytokines and chemokines. The IL-33 
Table I. The primer sequences used for PCR.

\begin{tabular}{|c|c|c|}
\hline $\begin{array}{l}\text { Gene } \\
\text { name }\end{array}$ & Primer & Sequence $\left(5^{\prime}-3^{\prime}\right)$ \\
\hline \multirow[t]{2}{*}{ IL-6 } & Forward & АСТCACСТCTTCAGAACGAATTG \\
\hline & Reverse & CCATCTTTGGAAGGTTCAGGTTG \\
\hline \multirow[t]{2}{*}{ IL-8 } & Forward & TTTTGCCAAGGAGTGCTAAAGA \\
\hline & Reverse & AACCCTCTGCACCCAGTTTTC \\
\hline \multirow[t]{2}{*}{ MIG } & Forward & CCAGTAGTGAGAAAGGGTCGC \\
\hline & Reverse & AGGGCTTGGGGCAAATTGTT \\
\hline \multirow[t]{2}{*}{ IP-10 } & Forward & GTGGCATTCAAGGAGTACCTC \\
\hline & Reverse & TGATGGCCTTCGATTCTGGATT \\
\hline \multirow[t]{2}{*}{ IL-1 $\beta$} & Forward & CCACCTCCAGGGACAGGATA \\
\hline & Reverse & AACACGCAGGACAGGTACAG \\
\hline \multirow[t]{2}{*}{ TNF- $\alpha$} & Forward & GAGGCCAAGCCCTGGTATG \\
\hline & Reverse & CGGGCCGATTGATCTCAGC \\
\hline \multirow[t]{2}{*}{ GAPDH } & Forward & GCACCGTCAAGGCTGAGAAC \\
\hline & Reverse & TGGTGAAGACGCCAGTGGA \\
\hline
\end{tabular}

concentration exhibited an inverted-U-shaped curve in response to IL-6, IL-1 $\beta$, IL-8, MIG and IP-10, but not TNF- $\alpha$, in synovial fluid of patients with RA (Fig. 2). The same IL-33 inverted-U-shaped association was observed after treatment with MIG but notIP-10, TNF- $\alpha$, IL- 6 , IL-1 $\beta$ or IL-8 in the serum of patients with RA (Fig. 3).

$m R N A$ and protein expression levels of $I L-6, I L-8, M I G, I P-10$, $I L-1 \beta$ and TNF- $\alpha$ in FLSs stimulated by $I L-33$. As presented in Fig. 4, IL-33 affected the mRNA expression of IL-6, IL-8, MIG, IP-10, IL- $1 \beta$ and TNF- $\alpha$ in FLS. However, only the protein expression of IL-6, IL-8, IP-10 and TNF- $\alpha$ was detectable in the supernatants of the FLS cell culture. IL-33 affected both the mRNA and protein expression of IL-6 in FLSs. A high response of FLSs at a concentration of $50 \mathrm{ng} / \mathrm{ml} \mathrm{IL-33}$ suggested that it had a narrow working concentration on FLSs (Fig. 4A). A greater response at $4 \mathrm{~h}$ than at $24 \mathrm{~h}$ suggested that IL-33 is effective over a precise time frame in FLSs. Over time, the effect of IL-33 on IL- 6 secretion by FLSs changed (Fig. 4B). Different to IL-6, the expression of IL-8 reached a peak after $24 \mathrm{~h}$ at a concentration of $50 \mathrm{ng} / \mathrm{ml}$ of IL-33 (Fig. 4C and D). MIG mRNA expression was upregulated in the 50 and $100 \mathrm{ng}$ groups after $4 \mathrm{~h}$, but only in the $50 \mathrm{ng}$ group after $24 \mathrm{~h}$ (Fig. 4E), while MIG protein could not be detected in the FLS culture supernatant. IL-6, IL-8 and IL-1 $\beta$ mRNA were detected at high levels in both the 50 and $100 \mathrm{ng}$ IL-33 groups at both time-points, but MIG and IL-1 $\beta$ protein expression was not detected in the supernatants of FLSs, even at low levels. (Fig. 4F). IP-10 mRNA expression was downregulated as the concentration of IL-33 increased (Fig. 4G). The ELISA results also demonstrated that the protein expression of IP-10 decreased as the concentration of IL-33 increased after $24 \mathrm{~h}$. Conversely, TNF- $\alpha$ and IL-17 increased the IP-10 mRNA expression in FLSs (Fig. 4H). TNF- $\alpha$ protein and mRNA expression was affected by different concentrations of IL-33 or by IL-17, but the obvious inverted-U-shaped regularity was not observed. (Fig. 4I and J).

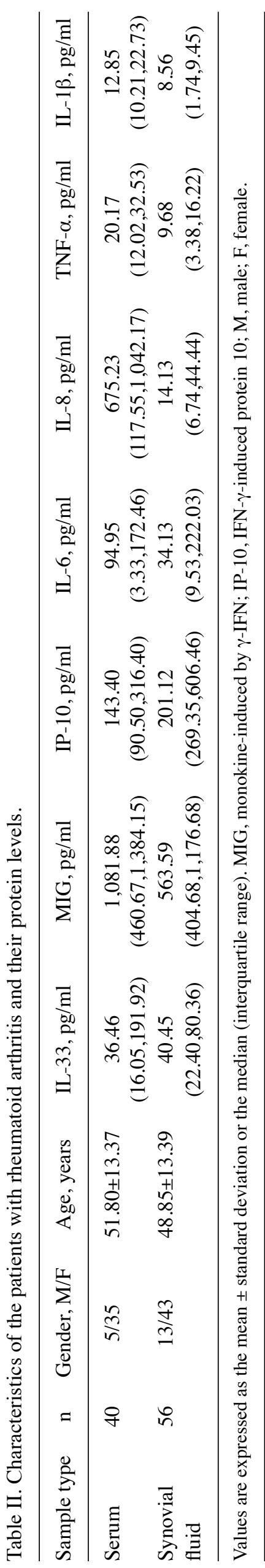


A

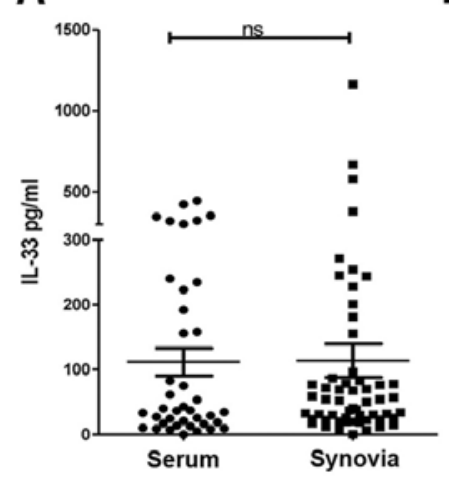

B

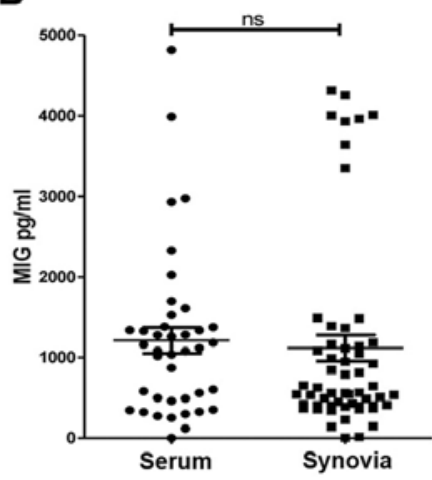

C

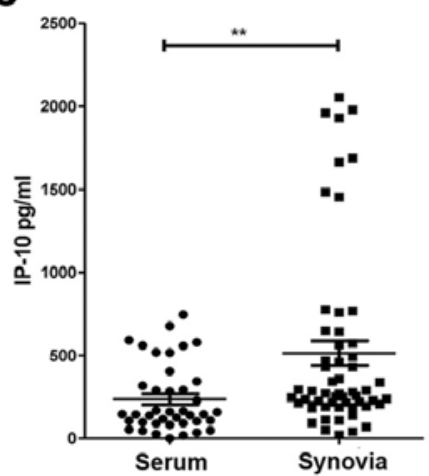

D

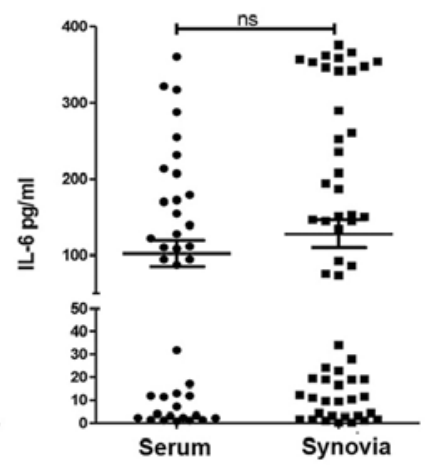

E

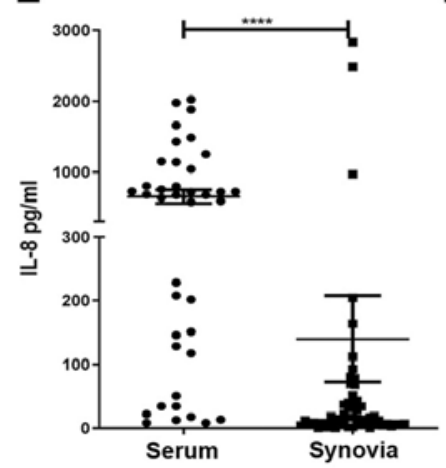

$\mathbf{F}$

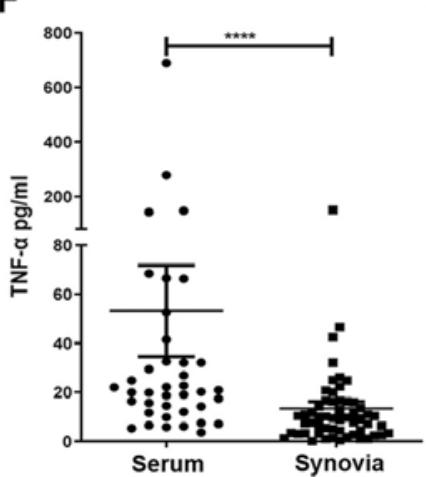

G

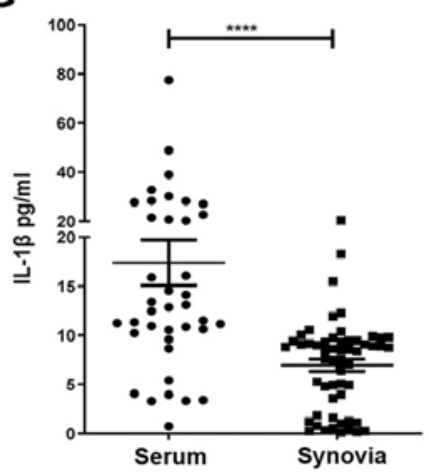

Figure 1. Concentration of cytokines and chemokines in serum and synovia, measured by ELISA, were obtained from 96 patients with RA ( $\mathrm{n}=40$ serum and 56 synovial samples). (A) IL-33 (P=0.640), (B) MIG (P=0.183), (C) IP-10 (P=0.001), (D) IL-6 (P=0.376), (E) IL-8 (P<0.001), (F) TNF- $\alpha$ (P<0.001) and (G) IL-1 $\beta(\mathrm{P}<0.001)$ levels were compared between RA synovia and serum. ${ }^{* *} \mathrm{P}<0.01,{ }^{* * * *} \mathrm{P}<0.001$ as assessed by the Mann-Whitney $\mathrm{U}$-test, as appropriate. Ns, no significance; RA, rheumatoid arthritis; MIG, monokine-induced by $\gamma$-IFN; IP-10, IFN- $\gamma$-induced protein 10 .

\section{Discussion}

Previous studies have indicated that IL-33 is expressed in the synovium of patients with RA, promoting articular inflammation $(12,15,20)$; however, the underlying mechanisms have not been fully elucidated and conflicting experimental results have been observed on numerous occasions. In vivo, injection of IL-33 has been demonstrated to exacerbate joint inflammation in a $\mathrm{K} / \mathrm{BxN}$ serum-transfer mouse model of arthritis (17). However, a separate study provided conflicting results, indicating that injection of IL-33 ameliorated joint inflammation in the same mouse model (18). Of note, another two research groups observed similar contradictory results: Biton et al (25) reported that IL-33 was able to suppress inflammation in collagen-induced arthritis (CIA) mice, but Xu et al (12) demonstrated that IL-33 was a critical proinflammatory cytokine causing fibroblast activation in CIA mice. The contradictory results may be due to the different dosage of IL-33 injected. Therefore, whether the effect of IL-33 was proinflammatory or inhibitory may be dependent on its concentration.

To confirm this hypothesis, an additional series of experiments was performed. First, the concentrations of a variety of cytokines and chemokines were measured in synovia and serum. Of note, the level of IP-10 was considerably higher in synovial fluid than in serum, indicating that inflamed joints constitute the primary source of circulating IP-10 in arthritis. Conversely, IL-8, TNF- $\alpha$ and IL-1 $\beta$ levels were substantially higher in serum than in synovial fluid. Next, the association of
IL-33 with other cytokines and chemokines in patients with RA was investigated. As expected, IL-33 was not linearly correlated with other cytokines and chemokines but exhibited an inverted-U-shaped association. In the present study, IL-33 exhibited an inverted-U-shaped association with IL-6, IL-8, IL-1 $\beta$, MIG and IP-10 in the synovia of patients with RA. Although the results may reveal the mechanism of IL-33 in RA, in numerous instances, the data points at the right side of the $\mathrm{x}$-axis do not map onto the U-shaped fit, hence, U-shaped model may only have limited applicability in clinical practice. In the cell model, there appears to be some U-shaped effects of IL-33. This notion may indeed be valid, but the mathematical U-shaped curve fit does not seem ideal in serum and synovial fluid samples for the patient. This fact does make the U-shaped association insignificant. Therefore, the sample size needs to be expanded to increase the validity of the results and the conclusions of the present study. Although there is an inverted-U-shaped association between synovial IL-33 and MIG, IP-10, IL-6, IL-8, IL-1 $\beta$ in patients with RA, it may be disproportionately influenced by only a couple of data points as high concentration points, which limited the extrapolation of conclusions of the present study. Therefore, it is of note that in the present study, the datapoints do not exactly follow the U-shaped curve fit in most cases. Therefore, the applicability of this mathematical model in clinical practice is limited.

Although the conclusions are limited, the study partially illustrates that IL-33 may have a greater effect on synovial cells and that IL-33 has a more specific role in synovia than 

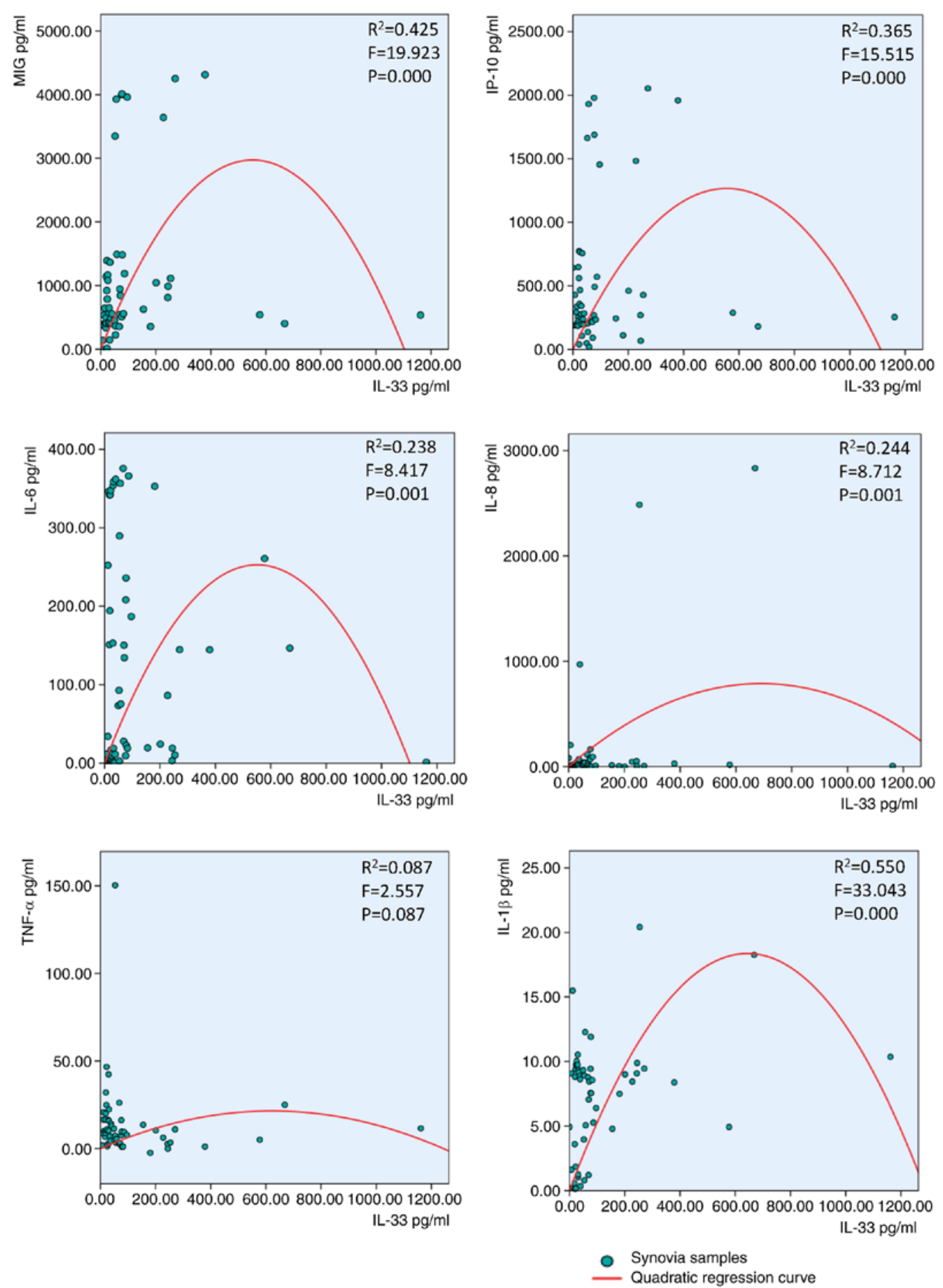

Figure 2. Inverted-U-shaped association between synovial IL-33 and MIG, IP-10, IL-6, IL-8, TNF- $\alpha$ and IL-1 $\beta$ in patients with rheumatoid arthritis. Second-order polynomial regression curves were fitted with SPSS 24.0. MIG, monokine-induced by $\gamma$-IFN; IP-10, IFN- $\gamma$-induced protein 10.

in serum, since this U-shaped association occurs only in synovia. Synovia is principally produced by synovial cells, and therefore, the present study aimed to explore the role of IL-33 in synovial cells. The experiment was designed with various IL-33 concentrations stimulating FLSs. As expected, the most noteworthy result was that specific concentrations of IL-33 were important in FLS cultures, with $10 \mathrm{ng} / \mathrm{ml}$ being too low to cause an impact on FLS but $150 \mathrm{ng} / \mathrm{ml}$ IL-33 being too high. This response was consistent with the U-type association between IL-33 and other cytokines observed in synovial fluid.
The most appropriate concentration of IL-33 was $50 \mathrm{ng} / \mathrm{ml}$ for 20,000 FLSs, although its influence was not comparable with IL-17 and TNF- $\alpha$, which strongly influenced FLSs. In another study, negative results were obtained (26), which may be because IL-33 was effective within a narrow range of working concentrations and the most appropriate ratio of the IL-33 concentration/cell number was important in FLS cultures. Time-points were an additional factor that affected FLS, with the incubation time of $4 \mathrm{~h}$ being more effective than $24 \mathrm{~h}$ regarding IL- 6 release, suggesting that IL-33 affects 

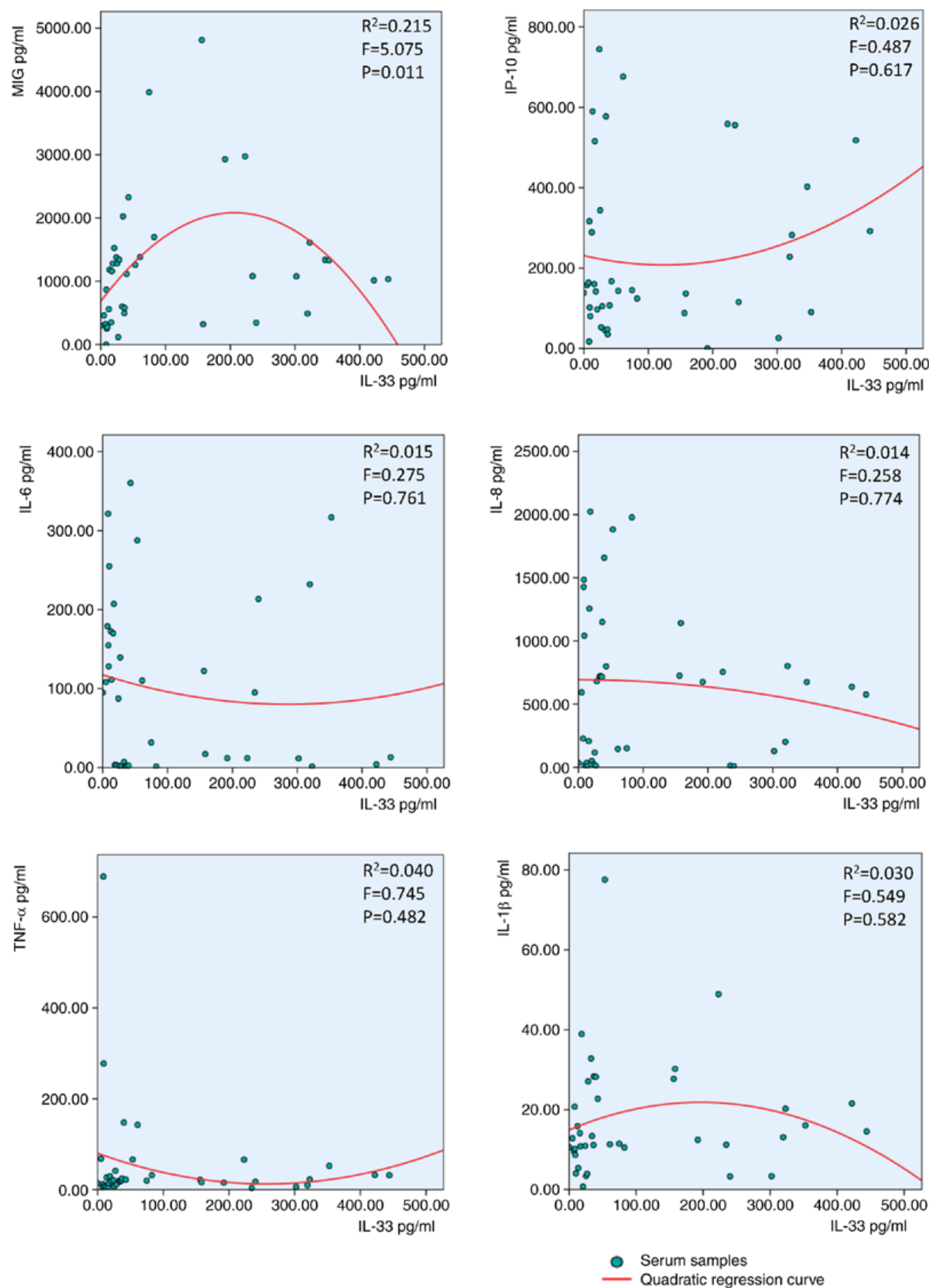

Figure 3. Inverted-U-shaped association between serum IL-33 and MIG, IP-10, IL-6, IL-8, TNF- $\alpha$ and IL-1 $\beta$. Second-order polynomial regression curves were fitted by SPSS 24.0. MIG, monokine-induced by $\gamma$-IFN; IP-10, IFN- $\gamma$-induced protein 10 .

FLS over a precise time frame. IL-6 was released earlier than IL-8, suggesting that different cytokines released by FLSs were controlled by different mechanisms. A significant inverted-U-shaped influence on IL-6 and IL-8, but not IP-10 and TNF- $\alpha$, was obtained, and therefore, the results imply that different cytokines released by FLSs were controlled by different mechanisms. IL-33 regulated the production of IL-1 $\beta$ in FLS in an inverted U-shaped manner and combined with the results of another study, which established that IL- $1 \beta$ regulated the production of IL-33 in FLS (27), it was demonstrated that IL-33 has a key role in the positive and negative feedback mechanisms with IL-1 $\beta$. In the present study, the association between IL-33 and other cytokines and chemokines was assessed. Initially, the inverted-U-shaped association between IL-33 and the chemokines IL-8, MIG and IP-10 in synovia was shown. These results are important for RA, suggesting that IL-33 has the potential capability to recruit and regulate inflammatory cells. A limitation of the present study was that although the U-shaped fit used appeared to be the best-fitting curve for the present data, the insufficient number 

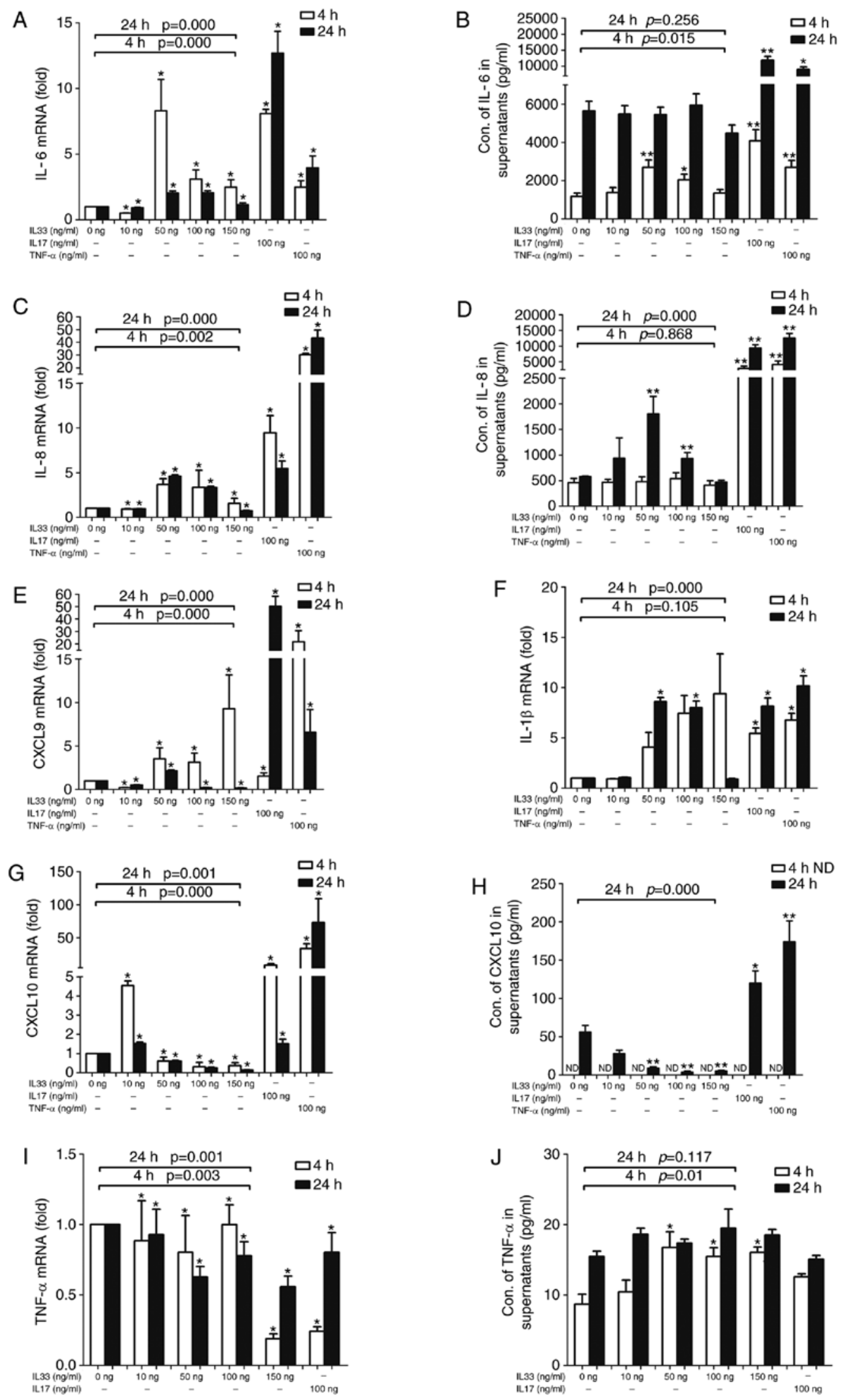

Figure 4. Effects of IL-33 on RA-FLSs. The expression levels of cytokines and chemokines by FLSs, stimulated by different concentrations of IL-33 for different durations, were analyzed using reverse transcription-quantitative PCR, and the amount of cytokines and chemokines released into the cell culture supernatant was determined using ELISA. (A) The expression levels of IL-6 mRNA in FLS. (B) The concentration of IL-6 in supernatant. (C) The expression levels of IL-8 mRNA in FLS. (D) The concentration of IL-8 in supernatant. (E) The expression levels of MIG mRNA in FLS. (F) The expression levels of IL-1 $\beta$ mRNA in FLS. (G) The expression levels of IP-10 mRNA in FLS. (H) The concentration of IP-10 in supernatant. (I) The expression levels of TNF- $\alpha$ mRNA in FLS. (J) The concentration of TNF- $\alpha$ in supernatant. $n=5$. Each experiment was repeated three times). ${ }^{*} \mathrm{P}<0.05$ and ${ }^{* *} \mathrm{P}<0.01$ vs. control group. The Mann-Whitney U-test was used as a post hoc test after the Kruskal-Wallis test for the different IL-33 concentration groups. Bonferroni's correction was performed for these data. ND, not determined; RA, rheumatoid arthritis; FLS, fibroblast-like synoviocytes. 
of high-concentration values of IL-33 limited the extrapolation of conclusions. In the future, more high-concentration value data of IL-33 will be added to strengthen the current conclusions. In clinical testing, high-concentration value samples for IL-33 are relatively rare; however, increasing the sample size may be a suitable solution.

The present study reported that IL-33, a member of the IL-1 family of cytokines, exhibits an inverted-U-shaped association with multiple cytokines and chemokines in synovial fluid, including IL-6, IL-1 $\beta$, IL-8, MIG and IP-10, but not in serum. IL-33 also triggered the expression of the pro-inflammatory cytokines IL- 6 and IL- $1 \beta$ and the chemokines IL- 8 and MIG in a U-shaped dose-dependent manner in RA-FLSs.

\section{Acknowledgements}

Not applicable.

\section{Funding}

This study was supported by the National Natural Science Foundation of China (grant no. 81601397) and the National Natural Science Foundation of China (grant no. 81771727).

\section{Availability of data and materials}

The datasets used and/or analyzed during the present study are available from the corresponding author on reasonable request.

\section{Authors' contributions}

JW, QL and JXD performed the experiments. JW and QL conceived the study and analyzed the results. JJZ and QHY designed the experiments and revised the manuscript. All authors read and approved the final manuscript.

\section{Ethics approval and consent to participate}

The study was approved by the Ethics Committee of Zhujiang Hospital of Southern Medical University (Guangzhou, China; grant no. 2019-KY-091-01). This was to certify that the research design and methods are in accordance with the requirements of the ethical standards of the 2013 Declaration of Helsinki, and regulations and procedures regarding human subject protection in Chinese laws. Written informed consent was obtained from all study participants.

\section{Patient consent for publication}

Not applicable.

\section{Competing interests}

The authors declare that they have no competing interest.

\section{References}

1. Nanki T, Nagasaka K, Hayashida K, Saita Y and Miyasaka N: Chemokines regulate IL- 6 and IL- 8 production by fibroblast-like synoviocytes from patients with rheumatoid arthritis. J Immunol 167: 5381-5385, 2001
2. Szekanecz Z, Kim J and Koch AE: Chemokines and chemokine receptors in rheumatoid arthritis. Semin Immunol 15: 15-21, 2003.

3. Kuan WP, Tam LS, Wong CK, Ko FW, Li T, Zhu T and Li EK: CXCL 9 and CXCL 10 as sensitive markers of disease activity in patients with rheumatoid arthritis. J Rheumatol 37: 257-264, 2010.

4. Ruschpler P, Lorenz P, Eichler W, Koczan D, Hänel C, Scholz R, Melzer C, Thiesen HJ and Stiehl P: High CXCR3 expression in synovial mast cells associated with CXCL9 and CXCL10 expression in inflammatory synovial tissues of patients with rheumatoid arthritis. Arthritis Res Ther 5: R241-R252, 2003.

5. Pandya JM, Lundell AC, Andersson K, Nordstrom I, Theander E and Rudin A: Blood chemokine profile in untreated early rheumatoid arthritis: CXCL10 as a disease activity marker. Arthritis Res Ther 19: 20, 2017

6. Siebert S, Tsoukas A, Robertson J and McInnes I: Cytokines as therapeutic targets in rheumatoid arthritis and other inflammatory diseases. Pharmacol Rev 67: 280-309, 2015.

7. Brennan FM and McInnes IB: Evidence that cytokines play a role in rheumatoid arthritis. J Clin Invest 118: 3537-3545, 2008.

8. Hueber AJ, Asquith DL, McInnes IB and Miller AM: Embracing novel cytokines in RA-complexity grows as does opportunity! Best Pract Res Clin Rheumatol 24: 479-487, 2010.

9. Mateen S,Zafar A, Moin S, Khan AQ and Zubair S: Understanding the role of cytokines in the pathogenesis of rheumatoid arthritis. Clin Chim Acta 455: 161-171, 2016.

10. He R, Yin H, Yuan B, Liu T, Luo L, Huang P, Dai L and Zeng K: IL-33 improves wound healing through enhanced M2 macrophage polarization in diabetic mice. Mol Immunol 90: 42-49, 2017.

11. Matsuyama Y, Okazaki H, Tamemoto H, Kimura H, Kamata Y, Nagatani K, Nagashima T, Hayakawa M, Iwamoto $M$, Yoshio T, et al: Increased levels of interleukin 33 in sera and synovial fluid from patients with active rheumatoid arthritis. J Rheumatol 37: 18-25, 2010.

12. Xu D, Jiang HR, Kewin P, Li Y, Mu R, Fraser AR, Pitman N, Kurowska-Stolarska M, McKenzie AN, McInnes IB and Liew FY: IL-33 exacerbates antigen-induced arthritis by activating mast cells. Proc Natl Acad Sci USA 105: 10913-10918, 2008.

13. Rivellese F, Suurmond J, Habets K, Dorjée AL, Ramamoorthi N, Townsend MJ, de Paulis A, Marone G, Huizinga TW, Pitzalis C and Toes RE: Ability of interleukin-33- and immune complex-triggered activation of human mast cells to down-regulate monocyte-mediated immune responses. Arthritis Rheumatol 67: 2343-2353, 2015.

14. Pushparaj PN, Li D, Komai-Koma M, Guabiraba R, Alexander J, McSharry C and Xu D: Interleukin-33 exacerbates acute colitis via interleukin-4 in mice. Immunology 140: 70-77, 2013

15. Palmer G, Talabot-Ayer D, Lamacchia C, Toy D, Seemayer CA, Viatte S, Finckh A, Smith DE and Gabay C: Inhibition of interleukin-33 signaling attenuates the severity of experimental arthritis. Arthritis Rheum 60: 738-749, 2009.

16. Yuan FL, Li X, Lu WG, Li CW, Xu RS and Dong J: IL-33: A promising therapeutic target for rheumatoid arthritis? Expert Opin Ther Targets 15: 529-534, 2011.

17. Xu D, Jiang HR, Li Y, Pushparaj PN, Kurowska-Stolarska M, Leung BP, Mu R, Tay HK, McKenzie AN, McInnes IB, et al: IL-33 exacerbates autoantibody-induced arthritis. J Immunol 184: 2620-2626, 2010.

18. Anthony RM, Kobayashi T, Wermeling F and Ravetch JV: Intravenous gammaglobulin suppresses inflammation through a novel T(H)2 pathway. Nature 475: 110-113, 2011.

19. Mu R, Huang HQ, Li YH, Li C, Ye H and Li ZG: Elevated serum interleukin 33 is associated with autoantibody production in patients with rheumatoid arthritis. J Rheumatol 37: 2006-2013, 2010.

20. Talabot-Ayer D, McKee T, Gindre P, Bas S, Baeten DL, Gabay C and Palmer G: Distinct serum and synovial fluid interleukin (IL)-33 levels in rheumatoid arthritis, psoriatic arthritis and osteoarthritis. Joint Bone Spine 79: 32-37, 2012.

21. Aletaha D, Neogi T, Silman AJ, Funovits J, Felson DT, Bingham CO III, Birnbaum NS, Burmester GR, Bykerk VP, Cohen MD, et al: 2010 Rheumatoid arthritis classification criteria: An American College of Rheumatology/European League Against Rheumatism collaborative initiative. Arthritis Rheum 62: 2569-2581, 2010.

22. Izawa N, Hirose J, Fujii T, Oka H, Uehara K, Naito M, Matsumoto T, Tanaka S and Tohma S: The utility of 25-question geriatric locomotive function scale for evaluating functional ability and disease activity in Japanese rheumatoid arthritis patients: A cross-sectional study using NinJa database. Mod Rheumatol 29: 328-334, 2019. 
23. Wu J, Li Q, Jin L, Qu Y, Liang BB, Zhu XT, Du HY, Jie LG and Yu QH: Kirenol Inhibits the function and inflammation of fibroblast-like synoviocytes in rheumatoid arthritis in vitro and in vivo. Front Immunol 10: 1304, 2019.

24. Livak KJ and Schmittgen TD: Analysis of relative gene expression data using real-time quantitative PCR and the 2(-Delta Delta C(T)) method. Methods 25: 402-408, 2001.

25. Biton J, Khaleghparast Athari S, Thiolat A, Santinon F, Lemeiter D, Hervé R, Delavallée L, Levescot A, Roga S, Decker P, et al: In vivo expansion of activated Foxp3+ regulatory $\mathrm{T}$ cells and establishment of a type 2 immune response upon IL-33 treatment protect against experimental arthritis. J Immunol 197: 1708-1719, 2016
26. Machado CRL, Resende GG, Macedo RBV, Nascimento VC, Sliva TP, Kakehasi AM and Andrade MVM: AB0028 Fibroblast-like synoviocytes may not be the target of il-33 in the joint phisiopathology. Ann Rheum Dis 76 (Suppl 2): S1056, 2017.

27. Lee EJ, So MW, Hong S, Kim YG, Yoo B and Lee CK: Interleukin-33 acts as a transcriptional repressor and extracellular cytokine in fibroblast-like synoviocytes in patients with rheumatoid arthritis. Cytokine 77: 35-43, 2016. 